\title{
A study on the Evaluation of the Core Disinfection Facility in South Korea
}

\author{
Wooseog Jeong, Mi Young Kang, Su Yeon Han, Young Wook Kim, Yong-Sang Kim \\ Veterinary Drugs \& Biologics Division, Animal \& Plant Quarantine Agency(APQA) \\ Gimcheon-si, Gyeongsangbuk-do, South Korea \\ Wjeong@korea.kr
}

\section{Extended Abstract}

South Korea has had several outbreaks of foot and mouth disease (FMD) and HPAI. The most important factor of FMD and HPAI virus spreading is mechanical propagation by vehicle, which accounts for more than $80 \%$. Accordingly, South Korea has installed core disinfection facilities at regional bases, and all livestock-related vehicles are compulsorily disinfected. Therefore, it is very important to measure the disinfection effect of core disinfection facilities. However, until now, the experiment has been difficult because embryonic egg or cell culture has to be done, which is time consuming and can't treat large amounts and also can't be used in places other than the lab.

In order to overcome these problems, we used gene quantification method with real time-qPCR and rapid test kit. 100ul of $10^{7}$ Avian Influenza virus (in 1\% BSA and 1\% yeast extract) was dropped on a stainless steel disc carrier and then dried for 1 hour. The virus coated disc carriers were reacted with disinfectant and then vortexed in $5 \mathrm{ml}$ of neutralization solution $(10 \%$ FBS) to stop the reaction and recover the virus. And to isolate viruses that were not destroyed by disinfectant in solution, 100ul of the solution was added to the rapid test kit, and only the test line was cut out to separate RNA and used for gene quantification using real time qPCR. 200ul of the neutralization solution was used for embryonic egg inoculation for titer measurements and real time-qPCR for genetic quantification, respectively.

Titer 1 of embryonic egg inoculation was found to correspond to ct value 3 of real time qPCR. And also using the rapid test kit, it is possible to transport survived virus from core disinfection facility to the laboratory. 